\title{
Cell interactions in Wound Biofilm and in vitro Biofilm Revealed by Electron Microscopy
}

\author{
Binbin Deng ${ }^{1}$, Jackson W. Peck ${ }^{1}$, David W. McComb ${ }^{1}$, Subhadip Ghatak ${ }^{2}$, Shomita Steiner ${ }^{2}$, Piya \\ Ghatak $^{2}$ and Chandan K. Sen ${ }^{2}$ \\ 1. Center for Electron Microscopy and Analysis (CEMAS), College of Engineering, The Ohio State \\ University, Columbus, OH, USA \\ 2. Davis Heart \& Lung Research Institute and Comprehensive Wound Center, Department of Surgery, \\ The Ohio State University Wexner Medical Center, Columbus, OH, USA
}

Biofilms are aggregation of bacteria which are encapsulated in extracellular polymeric substance (EPS) and form complex structures. Biofilm associated chronic wound is a significant burden for patient and healthcare system [1-2]. Treatment of biofilm infection designed based on in vitro culture rarely promote the chronic wound healing successfully. The principal limitation of applying results from in vitro biofilm to clinical wound biofilm treatment is that the controlled culture condition cannot represent the real environment of wound biofilm. To understand the structural details of how bacteria survive and thrive in wound, and also compare the structural differences of wound biofilm and in vitro biofilm, we applied Electron Microscopy (EM) techniques in structural study of porcine wound biofilm and in vitro biofilm.

Wound biofilms were collected from porcine model with a clinically relevant mixed-species infection that established in our lab. Wound biofilm tissues were cut into $200 \mu \mathrm{m}$ thick sections. In vitro pseudomonas aeruginosa PAO1 biofilms were cultured in agar plate at $37{ }^{\circ} \mathrm{C}$ for 48 hours. Wound biofilm sections and in vitro biofilm discs were chemically fixed and en bloc stained. After dehydration and infiltration, samples were embedded in durcupan resin and incubated at $60^{\circ} \mathrm{C}$ for 2 days. The resin embedded biofilm was processed by ultramicrotome. Electron microscopy data were collected on Helios Nanolab 600 DualBeam (FIB/SEM), Tecani F20 S/TEM, Probe Corrected Titan3 ${ }^{\mathrm{TM}}$ 80-300 S/TEM. Images were processed and visualized using IMOD [3], Chimera [4] and Avizo software packages.

Our EM result shows that wound biofilm seldom forms large aggregation on the surface of wound; instead bacteria actively interact with phagocytes, fibroblast, collagen and adipose cells (Figure 1). Bacteria niches in collagen and adipose tissues have various numbers of microorganism aggregation ranged from a few tens to a few hundreds. Occasionally isolated bacteria were found inside intact tissue, which may present a living state of bacteria that explore new niches and escape the chase of immunization cells. In vitro biofilm forms organized layers which can extend to $200 \mu \mathrm{m}$ thick. EPS between bacteria forms web-like intercellular networking (Figure 2) which may facilitate the nutrient and information transfer, promote biofilm grow and disperse. We concluded that bacteria and host interaction is the main scenario of wound biofilm. In vitro results can be applied to wound biofilm in limited local aggregation, but not to the whole wound environment. Effective wound biofilm treatment will need research work established on real wound model [5].

\section{References:}

[1] L Hall-Stoodley et al, Nature Reviews Microbiology 2(2) (2004), p. 95.

[2] T Bjarnsholt, APMIS suppl. 121(136) (2013), p. 1. 
[3] JR Kremer et al, Journal of Structural Biology 116(1) (1996), p. 71.

[4] EF Pettersen et al, Journal of Computational Chemistry 25(13) (2004), p. 1605.

[5] The authors acknowledge funding from Ohio Third Frontier Program, The Institute of Materials

Research and The Ohio State University
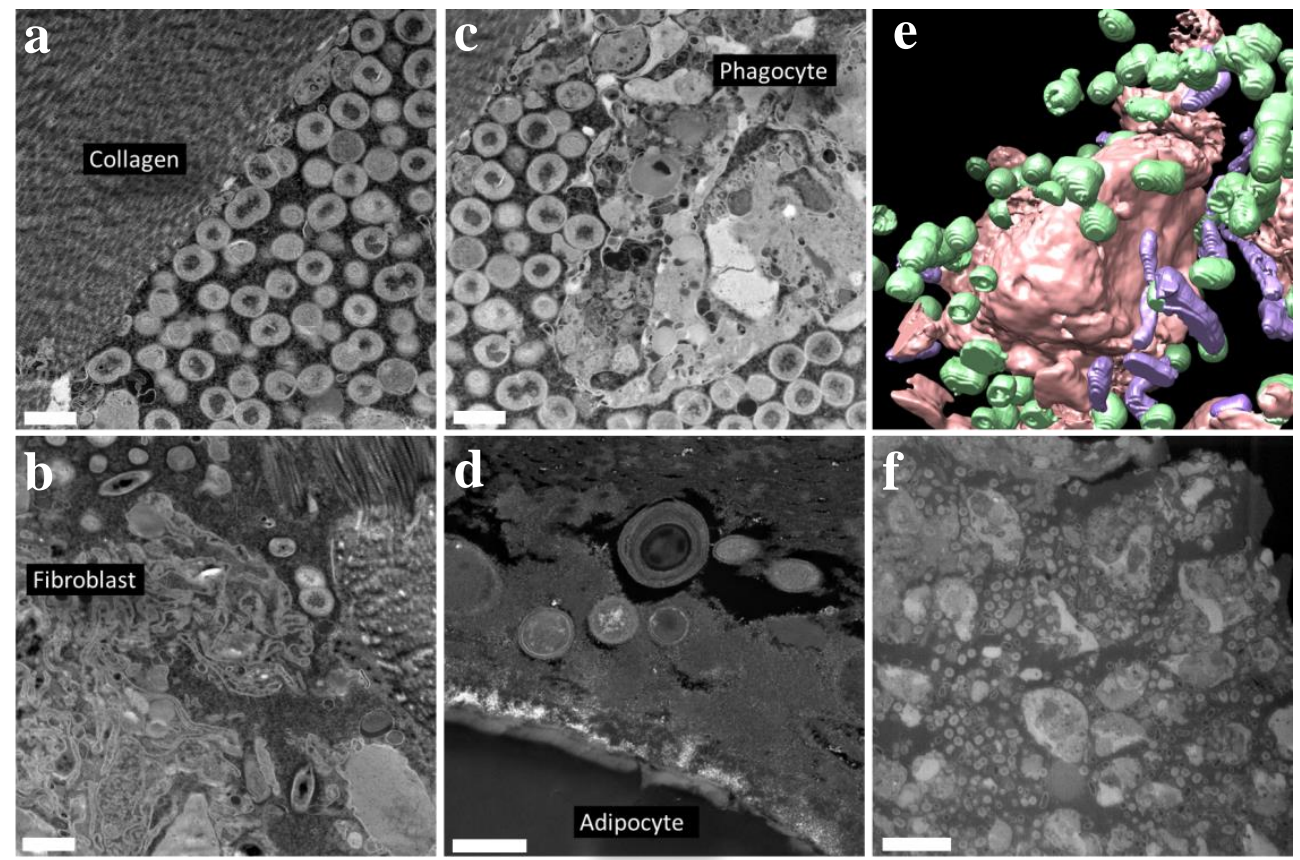

Figure 1. Cell interactions in wound biofilm. A. Bacteria and collagen. B. Bacteria and fibroblast; C Bacteria and phagocyte. C. Bacteria and adipocyte. E. Surface rendering of 3D structure of bacteria and fibroblast. Bacteria were colored in green and purple, fibroblast was colored in peach. F. Bacteria and adipocyte. Scale bar in a-d: $1 \mu \mathrm{m}$, in $\mathrm{f}: 5 \mu \mathrm{m}$.
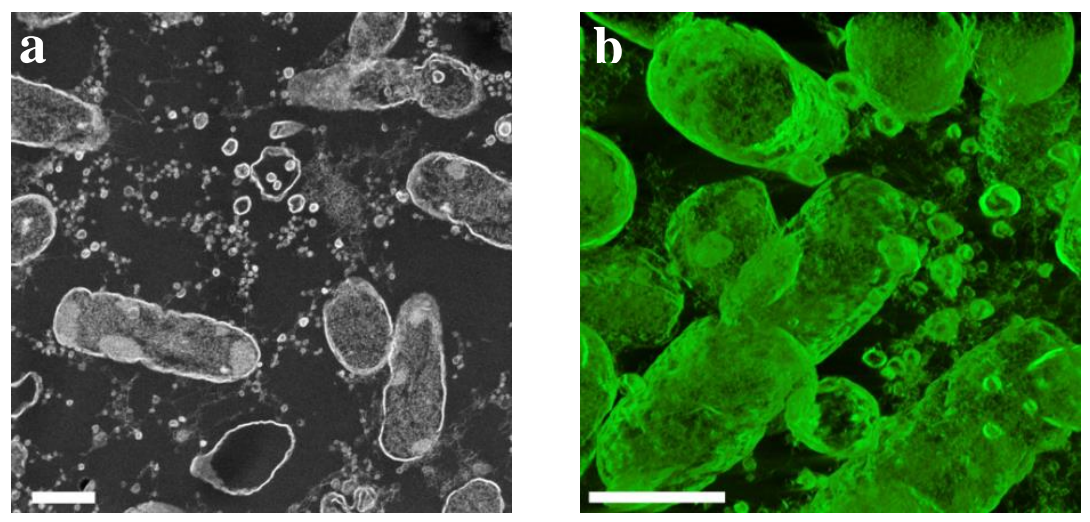

Figure 2. Extracellular Polymeric Substance (EPS) in in vitro Biofilm. A. STEM image of PAO1 biofilm. B. Volume rendering of PAO1 biofilm obtained from STEM tomography. Pseudo color was added. Scale bar: 500nm. 